\title{
A new parallel closing mechanism for the laminectomy rongeur makes it significantly more precise: a biomechanical and mechanical comparison study
}

\author{
Fabrice Alexander Külling ${ }^{1}$ Lukas Ebneter ${ }^{1}$ Georg Stefan Rempfler ${ }^{2}$ • \\ Vilijam Zdravkovic ${ }^{1}$
}

Received: 21 December 2016 / Revised: 20 September 2017 / Accepted: 8 October 2017 / Published online: 24 October 2017

(C) The Author(s) 2017. This article is an open access publication

\begin{abstract}
Purpose To prove that a modified closing mechanism of the rongeur gives better precision compared to the old Kerrison rongeur.

Methods Forty persons from the departments of orthopaedic surgery, urology and neurosurgery (35 orthopaedic, 2 urology and 3 neurosurgery) took part in the study. All participants were asked to punch ten times in a first step with either the old Kerrison rongeur with the scissors-like handle or the modified punch with a new parallel closing mechanism. In a second step, they punched 10 times with the other instrument. Shaft movement in three dimensions was measured with a stereoscopic, contactless, full-field digital image correlation system.

Results The new rongeur is significantly more precise with less movement in all three dimensions. The mechanical model of the new rongeur shows that the momentum needed to keep the tip at the initial position changes only minimally during the closing act on the new model.

Conclusion The new rongeur is more precise compared to the old Kerrison model. It is more robust against changes in the direction of the finger forces and may reduce soreness, fatigue and CTS in spine surgeons.

Level of evidence Not applicable: technical study.
\end{abstract}

Fabrice Alexander Külling

fabrice.kuelling@kssg.ch

1 Department of Orthopaedics and Traumatology, Kantonsspital St. Gallen, Rorschacherstrasse 95, 9007 St. Gallen, Switzerland

2 Swiss Federal Institute of Technology in Zurich (ETH), Zurich, Switzerland
Keywords Parallel closing mechanism $\cdot$ Rongeur · Laminectomy $\cdot$ Spine surgery $\cdot$ Accuracy $\cdot$ Decompression

\section{Introduction}

Neurosurgeons as well as orthopaedic spine surgeons use Kerrison rongeurs for decompression surgeries on the cervical, thoracic and lumbar spines. The Kerrison rongeur is used in about $80-100 \%$ of decompression surgery cases [1]. Together with the drill, it is probably the most commonly used instrument for this type of surgery. Dural lesions are the most common complications throughout spine surgery. In the lumbar spine surgery, it is reported with up to $17 \%$ in the literature [2,3]. In the thoracic spine, the incidence rate of cerebrospinal fluid leakage may be as high as $22 \%$ [4]. Incidental dural tears in the cervical spine are not as common as in the lumbar or thoracic spine and the incidence has been reported to range between 0.13 and 4\% [5-7]. Dural tears may lead to severe complications, including meningitis, arachnoiditis or fistula formation. Rheumatoid arthritis, age, deformities, length of time spent in OR, multiple level surgery and revision surgeries are all risk factors for an iatrogenic dural lesion. Even though preoperative imaging (MRI, CT scans) and improved operative techniques (MIS, microscope, loupes, navigation and head lamps) have been introduced over the last decade, the complication rate has not been reduced. Strömqvist et al. concluded in their register study that a dural lesion is a technical problem, which has to be solved during surgery [2]. It has also been reported that manual bone removal, when done over the course of several years, can lead to manual fatigue, tenderness, soreness in the hands and elbows, and as well as Carpal tunnel syndrome $[1,8]$. 
We analyzed the standard Kerrison rongeur and detected the high amplitude at the tip of the instrument while closing. In an operative field, where precision is mandatory, this effect may potentially lead to adverse events.

Taking a closer look at the closing mechanism of the standard Kerrison rongeur, the senior author (FK) developed a new parallel closing mechanism to address abovementioned problem.

The aim of this study was to analyze the difference between the closing mechanisms of the new rongeur and the old Kerrison rongeur and the effect to the amplitude of movement at the tip of the instrument in all three dimensions during several punching cycles. Furthermore, we compared the mechanical models of the two punches.

\section{Methods and materials}

Forty staff members from our hospital (ten residents, ten board certified surgeons, ten spine surgeons and ten office assistants) took part in this study. The spine surgeon group included only board certified orthopaedic surgeons and neurosurgeons with more than 4 years of spine experience. Primary outcome factor was the difference in amplitude at the tip of the instrument in all three dimensions $(x, y, z)$. According to a pilot study with five subjects, we performed a power analysis and determined that at least six subjects were needed to be included to show significance with $80 \%$ power.

\section{Measurements}

Shaft movement in the three axes $(x, y$, and $z)$ was measured in three dimensions with a stereoscopic, contactless, full-field digital image correlation system during opening and closing of the handles of the two different rongeurs (Vic-3D System, LIMESS, Correlated Solutions, Krefeld, Germany). The field of view was $100 \times 130 \mathrm{~mm}$ with accuracy of around 1 micron in the $x$, and $y$ planes, and around 2 microns in the $z$ plane. The punch frequency was according to a metronome with a frequency of 50 beats per min. During a sequence of ten punches according to the metronome tact, we captured three cycles (open/close) with each rongeur.

We defined the three planes as follows: $x$ plane was the extension of the shaft and therefore measured the forward/ backward movement of the rongeur. $y$ axis measured the up/down movement and $z$ plane measured the left/right movement.

The purpose of the study was explained to all participants. The subjects were standing in front of a table at the height of an OR table holding the rongeur in their dominant hand. To simulate a one-handed punch during cervical spine surgery, the participants were not allowed to stabilize their bodies on the table or hold the shaft of the rongeur with two hands. They aimed the tip of the rongeur towards a white cross without touching it. The shaft was at a $45^{\circ}$ angle to the table. We installed a wall of $\mathrm{Lego}^{\mathrm{TM}}$ bricks with a yellow $45^{\circ}$ line so that the study subjects could align themselves with it (Fig. 1). We randomized which rongeur was used first and allowed the subjects to punch ten times with each rongeur prior to the test to get used to the instruments.

Age, gender, and dominant hand and glove sizes were all recorded and analyzed.

\section{Mechanical model}

We performed a mechanical analysis of the closing mechanisms of the two rongeurs to show the different force diagrams. The traditional closing mechanism of the Kerrison rongeur works like scissors. The handles are hinged to the frame and by closing them the tip of the instrument closes. In between the handles is a return spring, which opens the rongeur as soon as you let go of the handles.

In the new rongeur, the scissors mechanism is replaced by a parallel handle bar which leads to a higher input/output force ratio. By pressing the handle, the tip of the instrument closes as well. We placed the identical return spring as in the conventional Kerrison rongeur to reopen the instrument. Figure 2 shows the mechanical model for both rongeurs.

The hand holding the punch is modelled by a rotational joint, a moment $M_{\mathrm{H}}$ controlling the rotation of the punch and two finger forces $\boldsymbol{F}_{\mathrm{H} i}(i=1,2$, and 5) pressing together the handles. The rotational joint fixes a point of the punch in space. The finger forces are modelled to be equally strong

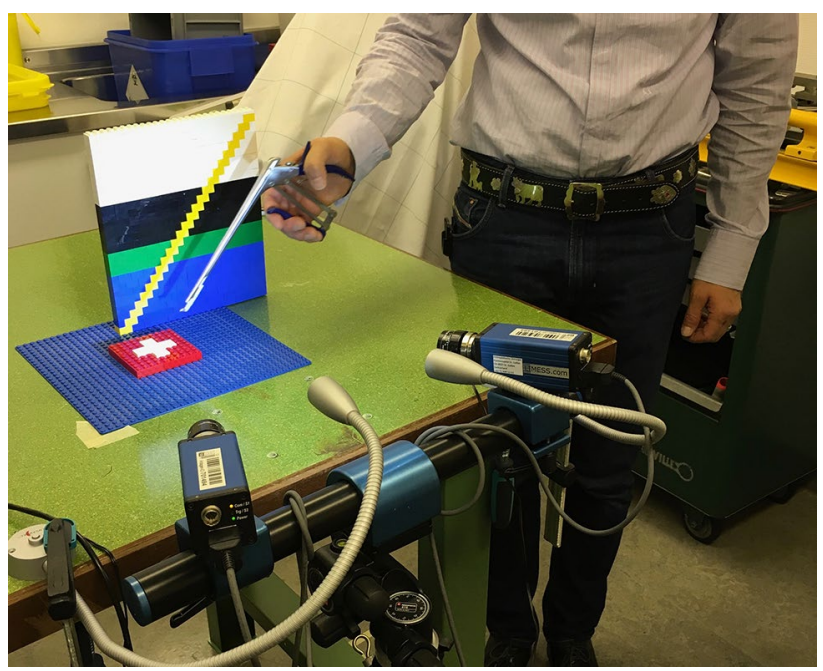

Fig. 1 Setting of the experiment. The wall of $\mathrm{Lego}^{\mathrm{TM}}$ at the back with the $45^{\circ}$ line in yellow. The participant had to aim toward the white cross without touching it. In the front, Vic-3D System, LIMESS, correlated solutions with two cameras 
Fig. 2 Showing the two mechanical models. On top, the traditional Kerrison rongeur and below the new model with the parallel handgrip. $\boldsymbol{F}_{\mathrm{H} i}$

$(i=1,2,5)$ model forces applied by the hand, $M_{\mathrm{H}}$ denotes a momentum applied by the hand. The angle $\beta$ defines how far the rongeur is closed. The angle $\alpha$ denotes the inclination of the rongeur
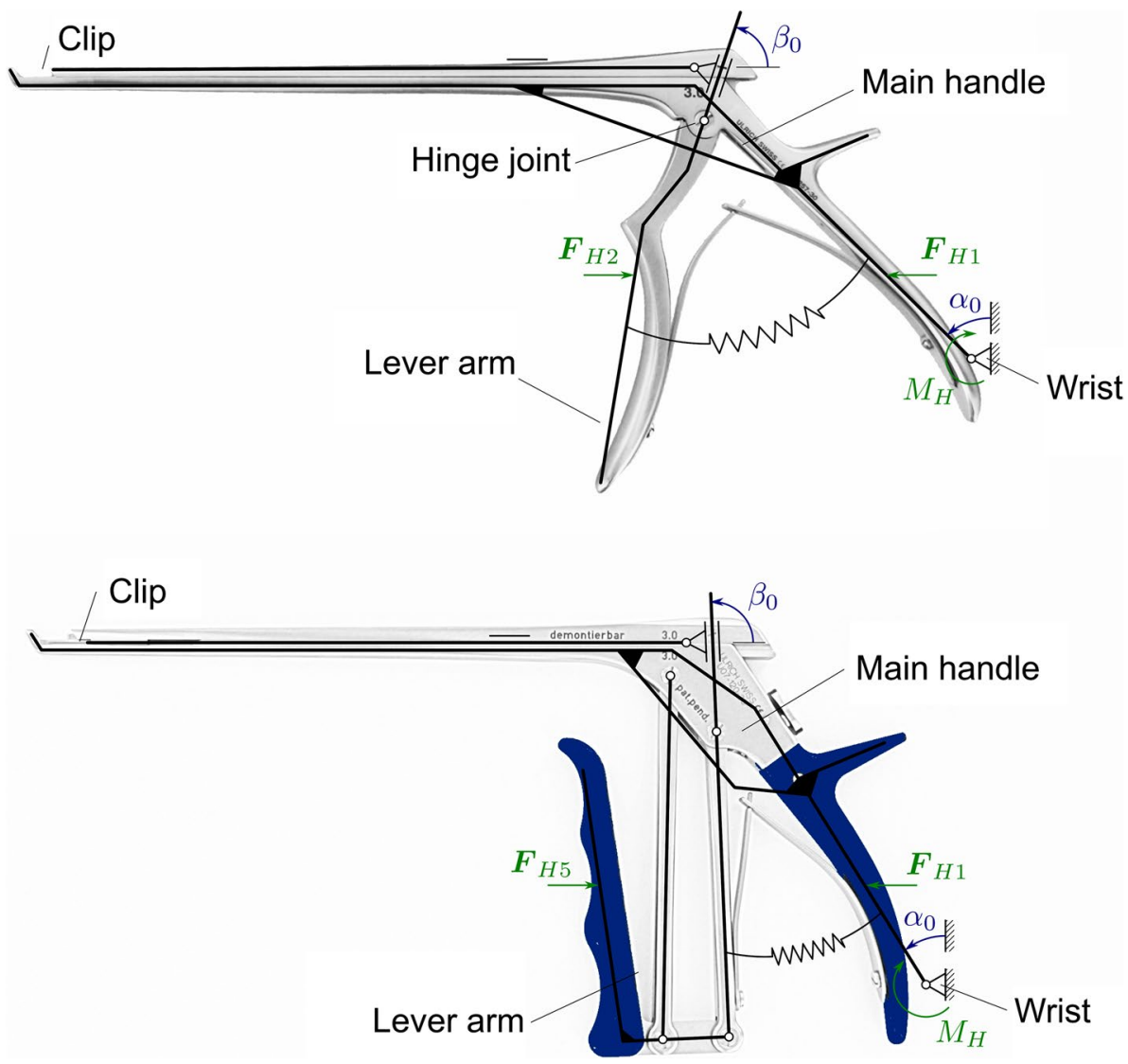

and to always point in horizontal direction. Over the range of all possible angles $\beta$, from the open $\left(\beta=\beta_{0}\right)$ to a fully closed punch, it is evaluated what moment $M_{\mathrm{H}}$ is needed to keep the tip of the punch at the initial position.

\section{Statistics}

All statistical analyses were performed with R (R: A language and environment for statistical computing. R Foundation for Statistical Computing, Vienna, Austria. URL http://www.R-project.org/). Descriptive statistics included means, standard deviations, ranges and proportions. We applied $t$ test and Mann-Whitney $U$ test for respective parametrical and non-parametrical bivariate analyses. The confidence level for rejecting null hypotheses was set at $95 \%$ $(p$ value $<0.05)$.

\section{Results}

A total of 14 females and 26 males were recruited for the study. In all 40 participants, the right hand was the dominant one.

The details of each group are shown in Table 1.

The average movement in the $x$ plane (forward/backward) was $5.8 \mathrm{~mm}$ for the new rongeur and $7.2 \mathrm{~mm}$ for the old Kerrison rongeur $(p=0.01)$. The up/down movement was measured on the $y$ plane and was significantly different between the two types. With the new rongeur, the amplitude was only $7.9 \mathrm{~mm}$ compared to $18.4 \mathrm{~mm}$ with the old model
Table 1 The details of the participants regarding age, glove size and dominant hand

\begin{tabular}{lllll}
\hline & Office assistants & Residents & Consultants & Spine surgeons \\
\hline Gender f/m & $8 / 2$ & $3 / 7$ & $3 / 7$ & $0 / 10$ \\
Mean age in years & 42 & 31 & 39 & 44 \\
Glove size: average & 7 & 7.5 & 8 & 8 \\
Dominant hand & $10 / 0$ & $10 / 0$ & $10 / 0$ & $10 / 0$ \\
Right/left & & & & \\
\hline
\end{tabular}


$\left(p=2.145 \times 10^{-15}\right)$. The $z$ plane (left/right deviation) was also significantly better with the new rongeur (Fig. 3) with an average of 11.1 versus $14.6 \mathrm{~mm}(p=0.00015)$.

By analyzing the mechanical model and solving the equations for the momentum $M_{H}$, it is shown that in the traditional Kerrison rongeur (Fig. 4, blue) the momentum increases by closing the scissors-like closing mechanism. In the new model (Fig. 4, red), the momentum decreases while closing the handles. The longer lever arm $s$ allows for a smaller range of angle $\beta$, i.e. approximately half the range of the Kerrison type. This leads to a shorter range of movement in the hand and therefore to higher accuracy. The longer lever arm leads to a higher input/output force ratio. For a given output (punch) force, less input is needed. Smaller forces and moments are realized.

In the first half of the range of angles $\beta$, the moment $M_{\mathrm{H}}$ stays around zero for the new rongeur. That means no user input is needed regarding the rotation of the punch or in other words the tip of the punch of the new rongeur is expected to remain approximately fixed within the first half of the closing process. This is not the case for the old rongeur where the moment is raised quickly, the user has to push down or hold the punch two handedly to neutralize the moment.

At the end of the experiment, we asked all participants which instrument they prefer. Almost all participants chose $(95 \%, n=35)$ the new model as it is easier to handle and needs less force to close it.

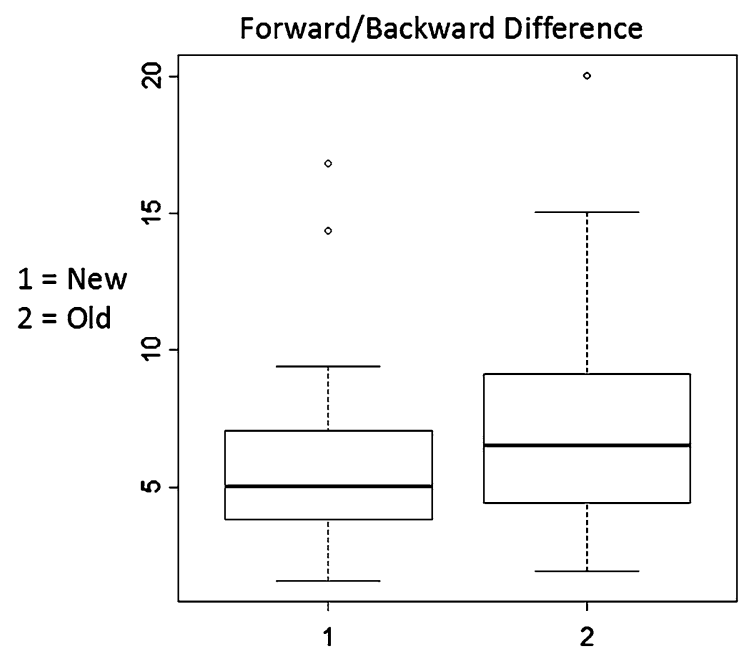

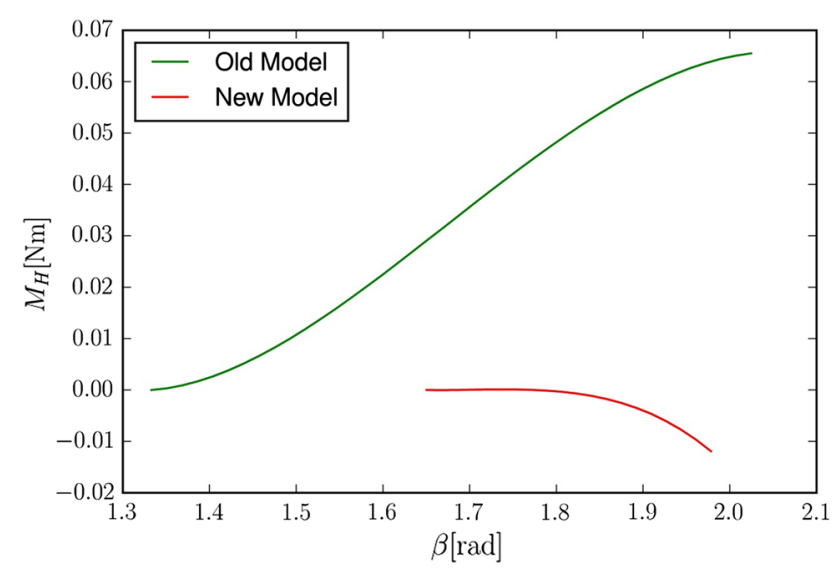

Fig. 4 For the moment $M_{\mathrm{H}}$, the following solutions were found in the Kerrison rongeur (old model in blue) and the new rongeur (new model in red)

\section{Discussion}

To our knowledge, this is the first study analyzing the force diagram and mechanical model of an instrument in daily use in spine surgery. While decompressing a spinal stenosis or performing a laminectomy or laminotomy, the surgeon works in close proximity to vital and vulnerable structures, such as the myelon, spinal nerves and blood vessels. Spine surgery relies upon exact fine motor skills to handle the neural elements as well as a steady hand over a long period of time. Therefore, not only is surgical dexterity important, but also the precision of the instruments is mandatory as they are the extensions of the surgeons' hands and fingers.

The handle of the new rongeur is designed so that the surgeon needs less force to close the mechanism and the

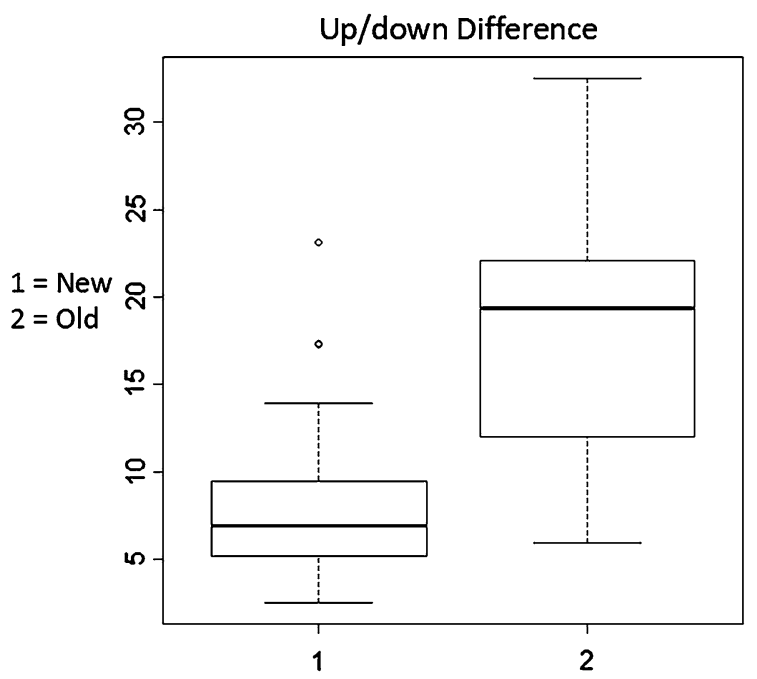

Fig. 3 Comparing the forward/backward as well as the up/down movement of the tip of the instrument, our data show that there is a significant reduction of amplitude in the new rongeur compared to the old one. This in the forward/backward as well as in the up/down movement 
momentum stays around zero. One needs less awareness of the rotational forces as the tip of the instrument remains in a fixed position during the closing and opening mechanism. This leads to a more precise movement and reduction of manual fatigue in the hands due to a better force diagram. Our results show that the new rongeur is more robust to counter changes in the direction of the finger forces $\boldsymbol{F}_{\mathrm{H} i}$. We were able to show that if the finger forces change direction the moment of the new rongeur remains similar, whereas in the old Kerrison rongeur the momentum increases rapidly. Therefore, it is easier to handle the new rongeur model.

These improvements in the new design of the handle and its mechanism lead to a more precise instrument performing a punching action. The new rongeur shows significantly better precision in the mechanical model, as well in the test series with 40 participants, where we could show significant reduction of instrument movement in all three dimensions. This may lead to a reduction of adverse effects during spinal surgery.

Forst et al. identified in their study the Kerrison rongeur as the greatest ergonomic risk factor for spine surgeons to suffer from Carpal tunnel syndrome (CTS) [8]. They stated that the Kerrison Rongeur should be evaluated ergonomically to reduce these injuries. The design of our new model requires the same tactile and proprioceptive sense we have acquired with the old Kerrison rongeur. Compared to the pneumatic Kerrison rongeur, there is no need for installation and additional training for the nurses. As in the pneumatic rongeur, our new model with the improvements in ergonomics of the handle and its mechanism may therefore reduce the rate of arthritis, carpal tunnel syndrome and pain in surgeon's hands and fingers, which are common problems for spine surgeons throughout their careers $[1,8]$.

Our study does have some limitations. We performed our test in an "in vitro" model outside of the operating room. While punching with the Kerrison rongeur, the instrument is often fixed against the bone or ligaments. This reduces the tip movement in most cases. In osteoporotic bone, the additional force against the bone may lead to adverse effects, such as dural tears or fractures.

Our idea was to simulate a one-handed removal of the posterior longitudinal ligament in the cervical spine or a decompression surgery in the lumbar spine using the other hand to hold the suction tube, as often performed by spine surgeons and described by Gunzburg et al. [9]. In this scenario, the surgeon needs a very steady instrument not only in the up/down but more importantly also in the forward/ backward movement. We were able to demonstrate especially in this scenario that the forward/backward movement of the new instrument is significantly reduced which might protect the myelon from a severe injury due to a hit. Even though the moving tip of the Rongeur is only one of many reasons for injuries, such as a Dural tear or a severe hit on the dura, the new closing mechanism hypothetically may lead to less injuries which have to be proven in a separate study.

In conclusion, we could demonstrate that the parallel closing mechanism of our new rongeur reduces the movement of the tip of the instrument significantly in all three dimensions due to fewer changes in the momentum of the instrument. With these little changes, the precision of the instrument could be raised significantly and it may reduce soreness, fatigue and CTS of the hand in spine surgeons.

\section{Compliance with ethical standards}

Conflict of interest The authors declares that they have no conflict of interest.

Open Access This article is distributed under the terms of the Creative Commons Attribution 4.0 International License (http://creativecommons.org/licenses/by/4.0/), which permits unrestricted use, distribution, and reproduction in any medium, provided you give appropriate credit to the original author(s) and the source, provide a link to the Creative Commons license, and indicate if changes were made.

\section{Appendix: mechanical model}

We drew the free-body diagrams for each body of the two models and stated the static equilibria equations for each body; a set of equations is obtained that fully describes all the unknown forces and moments in the systems.

See Fig. 5.

The corresponding equations for the free-body diagrams are

$$
\begin{aligned}
\mathbf{0}= & { }_{\mathrm{J}} \boldsymbol{A}+{ }_{\mathrm{J}} \boldsymbol{F}_{H 1}-{ }_{\mathrm{J}} \boldsymbol{B}-{ }_{\mathrm{J}} \boldsymbol{D}, \\
0= & \left({ }_{\mathrm{J}} \boldsymbol{a} \times{ }_{\mathrm{J}} \boldsymbol{A}\right)_{\mathrm{J}} \boldsymbol{e}_{z}^{\mathrm{J}}-M_{H}+\left({ }_{\mathrm{J}} \boldsymbol{f}_{1} \times \times_{\mathrm{J}} \boldsymbol{F}_{H 1}\right)_{\mathrm{J}} \boldsymbol{e}_{z}^{\mathrm{J}}+M_{R} \\
& -\left({ }_{\mathrm{J}} \boldsymbol{b}_{1} \times{ }_{\mathrm{J}} \boldsymbol{B}\right)_{\mathrm{J}} \boldsymbol{e}_{z}^{\mathrm{J}}-\left({ }_{\mathrm{J}} \boldsymbol{d}_{1} \times{ }_{\mathrm{J}} \boldsymbol{D}\right)_{\mathrm{J}} \boldsymbol{e}_{z}^{\mathrm{J}}-M_{D},
\end{aligned}
$$

for the first free-body diagram (old model) and

$$
\begin{aligned}
\mathbf{0}= & { }_{\mathrm{K}} \boldsymbol{Q}+{ }_{\mathrm{K}} \boldsymbol{B}-{ }_{\mathrm{K}} \boldsymbol{C}, \\
0= & -\left(q_{2 \mathrm{~K}} \boldsymbol{e}_{x}^{\mathrm{K}} \times_{\mathrm{K}} \boldsymbol{Q}\right)_{\mathrm{K}} \boldsymbol{e}_{z}^{\mathrm{K}}+\left(b_{2 \mathrm{~K}} \boldsymbol{e}_{x}^{\mathrm{K}} \times{ }_{\mathrm{K}} \boldsymbol{B}\right)_{\mathrm{K}} \boldsymbol{e}_{z}^{\mathrm{K}} \\
& -\left(\left(b_{2}+\frac{s}{\sin (\beta)}\right)_{\mathrm{K}} \boldsymbol{e}_{x}^{K} \times{ }_{K} \boldsymbol{C}\right)_{K} \boldsymbol{e}_{z}^{K}-M_{R},
\end{aligned}
$$

for the second free-body diagram (new model). These equations, together with the ones corresponding to bodies that are 

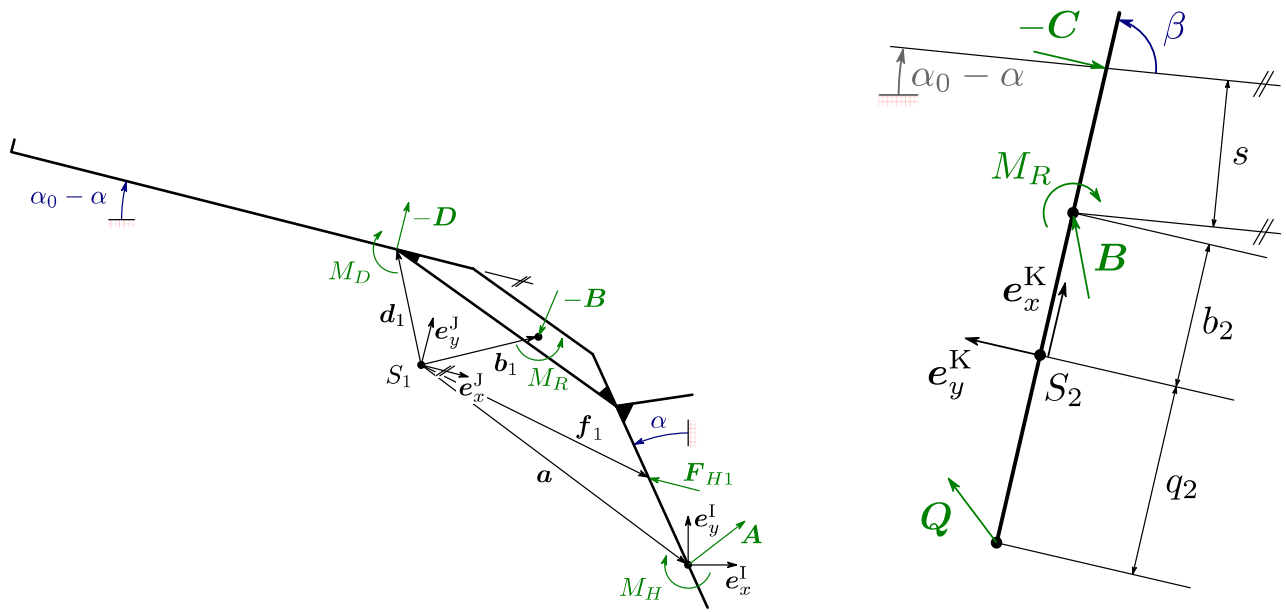

Fig. 5 Exemplarily, two free-body diagrams are presented. The first one is the main handle of the Kerrison rongeur, the second figure illustrates the rightmost bar of the handle mechanism of the new ron-

omitted here for brevity, are then numerically solved for all unknown forces and moments. Geometric values in the equations are determined from CAD drawings of the punches.

\section{References}

1. Maroon JC, El-Kadi M, Bost J (2009) A pneumatic Kerrison rongeur: technical note. Surg Neurol 71:466-468

2. Strömqvuist F, Jönsson B, Strömqvist B (2010) Dural lesions in lumbar disc herniation surgery: incidence, risk factors, and outcome. Eur Spine J 19:439-442

3. Herren C, Sobottke R, Mannion AF, Zweig T, Munting E, Otten P, Pigott T, Siewe J, Aghayev E (2017) Incidental durotomy in decompression for lumbar spinal stenosis: incidence, risk factors and effect on outcomes in the Spine Tango registry. Eur Spine J. doi:10.1007/s00586-017-5197-1

4. Xu N, Yu M, Liu X, Sun C, Chen Z, Liu Z (2017) A systematic review of complications in thoracic spine surgery for geur type. Items drawn in green represent forces and momenta acting on the (free cut) bodies. Vectors and scalars drawn in black denote geometric properties. The points $S_{1}$ and $S_{2}$ are the centres of mass

ossification of the posterior longitudinal ligament. Eur Spine J 26(7):1803-1809

5. Yoshihara H, Yoneoka D (2015) Incidental dural tear in cervical spine surgery. J Spinal Disord Tech 28(1):19-24

6. O'Neill KR, Neuman BJ, Peters C, Riew KD (2014) Risk factors for dural tears in the cervical spine. Spine 39(17):E1015-E1020

7. Fehlings MG, Smith JS, Kopjar B, Arnold PM, Yoon ST, Vaccaro AR et al (2012) Perioperative and delayed complications associated with the surgical treatment of cervical spondylotic myelopathy based on 302 patients from the AOSpine North America Cervical Spondylotic Myelopathy Study: clinical article. J Neurosurg Spine 16(5):425-432

8. Forst L, Fridman L, Shapiro D (2007) Carpal tunnel syndrome in spine surgeons: a pilot study. Arch Environ Occup Health 61(6):259-262

9. Gunzburg R, Szpalski M (2003) The conservative surgical treatment of lumbar spinal stenosis surgery in the elderly. Eur Spine J 12(Supp12):S176-S180 